\title{
Attention deficit/hyperactivity disorder's effects on individuals' scan paths during a simulated drive
}

\author{
J.R. Michaelis*, D.S. McConnell, and J. A. Smither \\ Technology \& Aging Laboratory, Psychology Department, University of Central Florida, 4000 Central Florida \\ Blvd., Orlando, FL 32816-1390, USA
}

\begin{abstract}
Individuals who have Attention Deficit/Hyperactivity Disorder (ADHD) experience adverse effects relating to driving; additionally, they experience deficits in scanning ability. The present study examined the effects of ADHD on eye tracking while driving. This study consisted of ten participants, of which, five have ADHD. It was hypothesized that individuals who have ADHD will make more saccadic eye movements and thus shorter fixations than individuals without ADHD (Control). Furthermore, it was hypothesized that despite the fact that individuals who have ADHD will make more saccadic eye movements than individuals without ADHD, those individuals with ADHD will commit more traffic violations including collisions compared to the control group. Findings indicated that hypothesis one was not supported by the data, whereas hypothesis two was supported in that ADHD individuals' had more collisions and committed more traffic violations than the control group. Additionally, a significant difference was found in the spatial distributions of the fixations between the ADHD and Control groups. The findings of this study could help better understand the factors involved in ADHD driving and could be used to train individuals with ADHD to become more aware of their surroundings and driving habits and thus become safer drivers.
\end{abstract}

Keywords: ADHD, Eye-tracking, Driving Simulator

\section{Introduction}

Attention Deficit Hyperactivity Disorder (ADHD) adversely affects driving. Individuals with ADHD experience deficits in scanning ability and as such have reduced fixations both in duration and frequency [4-6,8]. The present study examines the effects of ADHD on eye movements while driving. Attention deficit/hyperactivity disorder is a behavioral disorder characterized by persistent inattention, hyperactivity, and impulsiveness [1]. The symptoms as characterized by the disorder are very important to one's ability to drive safely. In fact it has been shown in previous research that individuals who have ADHD display riskier driving behaviors than nonADHD individuals, which include traffic violations such as frequent occurrences of speeding, lane devia- tions, and accidents, in addition to deficits in steering ability [5-6].

Despite the fact that impairments of varying degrees exist in drivers who have ADHD, no research has been conducted that has used an eye tracker to assess scanning and fixations in these individuals while driving in a simulator. However, research on scanning and ADHD has shown that individuals who have ADHD have deficits in response inhibition and as a result are less able to voluntarily inhibit unwanted saccades as well as a decreased ability to govern the amount and duration of fixations they make $[3,4,7,9]$. Additionally, individuals who have ADHD made significantly more large saccades during a visual fixation task than the control group [10].

Individuals with ADHD are expected to make more saccadic eye movements and thus shorter fixa-

\footnotetext{
* Corresponding Author. E-mail: jmichaelis@knights.ucf.edu
} 
tions than individuals without this disorder. Furthermore, individuals with ADHD should exhibit overall more saccadic eye movements than individuals without ADHD, and those individuals with ADHD will commit more traffic violations including collisions compared to the Control group.

\section{Methods}

\subsection{Participants}

The participants from a Southeastern University were comprised of 10 individuals, seven males and three females, of which, there were five Caucasians, two Hispanics, one Asian and one African American/Caucasian. They consisted of five individuals who did not have ADHD as well as five individuals registered with the Student Disability Services Office who had ADHD and were not currently taking medication for the disorder. Participants were required to have a valid driver's license, good vision, which was classified by at least having 20/40 vision with contacts or glasses, and not be prone to motion sickness based on the score of a motion history questionnaire.

Additionally, based on their self-reports, the number of hours spent driving in a typical week ranged from 1 to 21 hours and number of years that they were able to legally drive ranged from 2 to 14 years.

\subsection{Materials/ Apparatus}

A series of initial screening tools were implemented which consisted of the Motion History Questionnaire, the Simulator Sickness Questionnaire, an adult ADHD self-report symptom checklist (ASRS-v.1.1), the Driving Habits Questionnaire (DHQ), a demographics questionnaire, and a battery of vision tests. The battery of vision tests consisted of far and near visual acuity, color vision, and contrast sensitivity. Additionally, Multisoft's simulated driving software City Car Driving v.1.0, was used in conjunction with a Microsoft sidewinder steering wheel and pedals set up on a desk with the driving scenario projected on the wall in front of the participant. The projected scenario had a resolution of $1600 \times 1024$ and refresh rate of $60 \mathrm{~Hz}$. Furthermore, the simulator software also measured driving performance, including driving speed, lane deviations, and collisions. A daytime modern city scenario and motorway scenario with a moderate amount of traffic was used from the simulator's preexisting databank.
An ISCAN ETL-500 eye-tracker was used in conjunction with the driving software to record eye movements during the drive.

\subsection{Procedure}

Each participant was screened for vision deficits, $\mathrm{ADHD}$, and simulator sickness, and was given a driving history questionnaire. After which the eyetracker was calibrated to the participants and then they were given a 3 minute practice drive in a city scenario upon its conclusion they were given a 2 minute break. Participants were then asked to drive in the motorway scenario while wearing an ISCAN ETL-500 head mounted eye tracker which monitored the participants' saccades and fixations during the drive. Additionally, unsignaled lane deviations and collisions were recorded.

\section{Results}

Independent samples t-tests were conducted for both the duration and number of fixations made during the simulated motorway drive for both the ADHD and Control groups; however no significant differences were found.

Regardless of the fact that no significant differences were found for both the duration and number of fixations made, a Chi square test for independence suggested that there was a significant difference in spatial distributions of the fixations between the ADHD and control groups, $\chi^{2}(2, N)=10=26.22, p$ $<.01$, Cramer's $\mathrm{V}=.15$. This indicates that that there was an overall tendency to neglect the right visual field in both groups; however, the ADHD group looked to the right significantly less than the control group (Figure 1).

Furthermore, a Chi Square test for goodness of fit showed that there was a significant difference in the number of unsignaled lane deviations made by the ADHD and Control groups, $\chi^{2}(1, \mathrm{~N})=5, p<.05$ (Figure 2).

Finally, there was no statistical test to analyze the difference in accidents in the two groups. In total, four accidents were observed, all of which were committed by individuals in the ADHD group. 


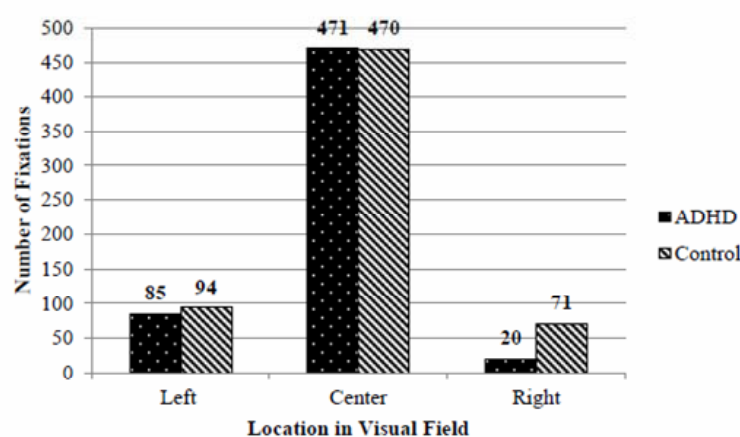

Fig. 1: Location of Fixations in the Visual Field

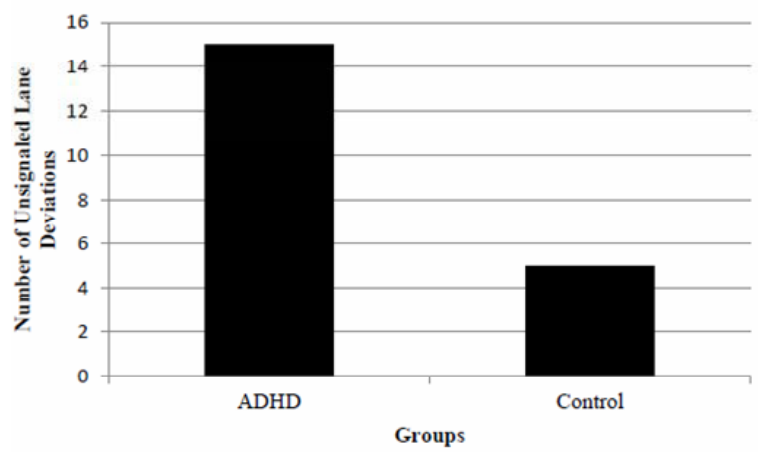

Fig. 2: Number of Unsignaled Lane Deviations

\section{Discussion}

The first hypothesis was not supported by the results which showed that both the ADHD and Control groups did not differ in the number of fixations made, and therefore, did not differ in number of saccades made as one is the inverse of the other. Furthermore, based on the assumption of the first hypothesis, it was assumed that the shorter fixation duration of the ADHD group would result in the ADHD group to incur more traffic violations and collisions. Despite the fact that the first hypothesis was not supported by the data, a difference was found for the number of unsignaled lane deviations and collisions.

Furthermore, a difference was found in the spatial distributions of the fixations between the two groups. Both groups neglected the right visual field, however, the control group fixated significantly more in the right visual field than the ADHD group. However, a small effect size was observed. That, and the lack of other significant differences in eye movements, may be attributed to the fact that a driving simulator was used. Previous research has shown that attention improves when individuals who have ADHD are placed in virtual reality environments [2]. Therefore, we might expect different results if eye movements were analyzed in a real world driving task. The findings of this study could help better understand the factors involved in ADHD driving, and could be used to train individuals with ADHD to become more aware of their surroundings and driving habits and thus become safer drivers.

\section{References}

[1] American Psychiatric Association, Diagnostic and statistical manual of mental disorders (Revised 4th ed.) Washington, DC, 2000, pp. 85-93.

[2] B. Cho et.al., The effect of virtual reality cognitive training for attention enhancement. CyberPsychology and Behavior 5 (2002), 129-137.

[3] C. Karatekin and R.F. Asarnow, Exploratory eye movements to pictures in childhood-onset schizophrenia and attention deficit/hyperactivity disorder (ADHD). Journal of Abnormal Psychology 27 (1999), 35-49.

[4] D.P., Munoz, I.T. Armstrong, K.A. Hampton, and K.D. Moore, Altered control of visual fixation and saccadic eye movements in attention-deficit hyperactivity disorder. Journal of Neurophysiology 90 (2003), 503-514.

[5] M. Fischer, R.A. Barkley, L. Smallish and K. Fletcher, Hyperactive children as young adults: Driving abilities, safe driving behavior, and adverse driving outcomes. Accident Analysis and Prevention 39 (2007), 94-105.

[6] R.A. Barkley, K.R. Murphy, and D. Kwasnik, Motor vehicle driving competencies and risks in teens and young adults with attention deficit hyperactivity disorder. Pediatrics 98 (1996), 1089-1095.

[7] R.G. Ross, J.G. Harris, A. Olincy, and A. Radant, Eye movement task measures inhibition and spatial working memory in adults with schizophrenia, ADHD, and a normal comparison group. Psychiatry Research 95 (2000), 35- 42.

[8] S. Nada-Raja, J.D. Langley, R. McGee, S.M. Williams, D.J. Begg and A.I. Reeder, Inattentive and hyperactive behaviors and driving offenses in adolescence. Journal of the American Academy of Child and Adolescent Psychiatry 36 (1997), 515522.

[9] S.H. Mostofsky, A.G. Lasker, L. Cutting, M.B. Denckla and D.S. Zee, Oculomotor abnormalities in attention deficit hyperactivity disorder: A preliminary study. Neurology 57 (2001), 423-430.

[10]T.D. Gould, T.M. Bastain, M.E. Israel, D.W. Hommer and F.X. Castellanos, Altered performance on an ocular fixation task in attention deficit/hyperactivity disorder, Society of Biological Psychiatry 50 (2001), 633-635. 